\title{
MAGE-A1/MAGE-A3/NY-ESO-1 Peptides Vaccine
}

National Cancer Institute

\section{Source}

National Cancer Institute. MAGE-A1/MAGE-A3/NY-ESO-1 Peptides Vaccine. NCI

Thesaurus. Code C84854.

A cancer vaccine comprised of synthetic peptides derived from human melanoma antigen A1 (MAGE-A1), human melanoma antigen A3 (MAGE-A3) and cancer-testis antigen NY-ESO-1 with potential immunostimulating and antineoplastic activities. Upon administration, MAGE-A1/MAGE-A3/NY-ESO-1 peptides vaccine may stimulate the immune system to mount a cytotoxic T-cell (CTL) response against tumor cells expressing MAGE-A1, MAGE-A3 and NY-ESO-1, resulting in tumor cell lysis. The MAGEA1, MAGE-A3, and NY-ESO-1 tumor-associated antigens (TAAS) are overexpressed by a variety of cancer cell types. 